\title{
Space qualification issues in acousto-optic and electro-optic devices
}

\author{
Narasimha S. Prasad ${ }^{1}$, Edward W. Taylor ${ }^{2}$, Sudhir Trivedi ${ }^{3}$, Sue Kutcher ${ }^{3}$ and Jolanta Soos ${ }^{3}$ \\ ${ }^{1}$ Narasimha S. Prasad, NASA Langley Research Center, Laser Remote Sensing Branch, \\ Hampton,Virginia 23681-2199 \\ ${ }^{2}$ International Photonics Consultants, 30 Tierra Monte NE, Albuquerque, NM 87122 \\ ${ }^{3}$ Brimrose Corporation, 19 Loveton Circle, Baltimore MD 21152-9201
}

\begin{abstract}
Satellite and space-based applications of photonic devices and systems require operational reliability in the harsh environment of space for extended periods of time. This in turn requires every component of the systems and their packaging to meet space qualifications. Acousto- and electro-optical devices form the major components of many current space based optical systems, which is the focus of this paper. The major space qualification issues are related to: mechanical stability, thermal effects and operation of the devices in the naturally occurring space radiation environment. This paper will discuss acousto- and electro-optic materials and devices with respect to their stability against mechanical vibrations, thermal cycling in operating and non-operating conditions and device responses to space ionizing and displacement radiation effects. Selection of suitable materials and packaging to meet space qualification criteria will also be discussed. Finally, a general roadmap for production and testing of acousto- and electro-optic devices will be discussed.
\end{abstract}

Keywords: Space qualification, acousto-optic devices, electro-optic devices, acousto-optics tunable filter, electro-optic modulator, radiation hardening.

\section{INTRODUCTION}

To be qualified for space missions, a device must be capable of withstanding extended operation in space and planetary environmental extremes. The extremes result from a combination of neutral ambient environment and spacecraft induced environments. These environments can be broadly categorized as (1) neutral gas, (2) plasma, (3) radiation (both electromagnetic and corpuscular), (4) particles (meteoroids and space debris) and (5) micro-gravity ${ }^{1,2}$. Instruments on board spacecraft must be able to withstand low temperatures, temperature cycling, high impact stresses, and radiation environments. In this paper, we discuss the space qualification issues for acousto-optic (AO) and electro-optic (EO) based devices.

Many AO-based devices, such as the acousto-optic tunable filter (AOTF) spectrometer and the acousto-optic modulator (AOM), are well suited for space environments. AOTF spectrometers can be powerful tools for the in-situ surface and subsurface chemical analysis of soils, rocks and ices ${ }^{3}$ as well as for astrobiology related experiments. AOTF is an electronically tunable optical filter in which optical beams passing through the AO crystal can be manipulated by frequency generated acoustic waves in an anisotropic crystal. Devices based on AOTF technology have been successfully used in space-based instruments where compact AOTF-based spectrometers and cameras are used for research and process control. JPL has developed an AOTF-based spectrometer for aerospace applications in which the AOTF device was reported to have undergone radiation hardness testing ${ }^{4}$. One of the first spectroscopic applications of an AOTF-based spectrometer used on a civilian spacecraft is in the SPICAM Light optical package ${ }^{5,6}$. This system is presently acquiring data on the ESA Mars Express mission. A stand-off, AOTF-based Raman imaging system that can be used for planetary measurements has also been reported ${ }^{7}$.

Of special interest are recent studies conducted and reported by Taylor and colleagues ${ }^{8-12}$ regarding radiationinduced effects and hardening of non-linear optical (NLO) polymer based modulators. While commercial polymer modulators are emerging, only a modicum of data has been reported for gauging the eventual space qualification of these 
devices. A discussion and brief examples of some key reported radiation induced responses in NLO polymer materials and modulator devices is presented at the end of this paper.

In the following paragraphs, we discuss the advantages of AO-based devices for space-based applications along with the issues that still need to be addressed.

\section{ADVANTAGES OF AOTF}

Space science missions increasingly require in-situ spectrometers for the characterization of the atmosphere, surface and subsurface regions of planets, satellites, and small bodies. A radio frequency (RF) signal drives the AOTF, which drives the transducer to generate sound waves inside the transducer. When a beam of broadband light travels through the filter, the filter deflects a thin slice of light from the whole spectrum. The line width of deflected energy can be as narrow as 1 to $2 \mathrm{~nm}$. The center wavelength of the spectral slice is determined by the RF frequency. By scanning the RF frequency, the whole wavelength spectrum can be obtained. AOTF has many features that are very attractive to the measurement of optical spectra in many adverse environments such as space ${ }^{13}$.

- AOTF is a rugged and compact device, with no moving Parts. AOTF is solid-state device and is both compact and rugged. This makes it suitable for planetary exploration missions. Just as important, short of actually breaking the device, vibrations and shocks will not affect the wavelength calibration or the alignment.

- AOTF naturally has two orthogonal polarization state outputs. AOTF can have two orthogonal linear polarization states as its outputs. Used with an achromatic waveplate, it is ideal for dichroism/birefringence measurements.

- AOTF has a built-in solid-state chopper which can serve as a lock-in amplifier. The intensity of the selected light is controlled electronically and can be rapidly modulated by changing the RF power that is supplied to the AOTF crystal. This makes the AOTF ideal for use with a lock-in (phase and frequency sensitive) amplifier for low-level light detection in the presence of strong ambient light.

- AOTF is extremely fast and can provide random access. The limiting factor for changing the wavelength is the time it takes for the acoustic wave to fill the optical aperture - typically several to several tens of a nanosecond. The entire spectra can be scanned at very high speed, or discrete wavelengths may be accessed at rates of tens to hundreds of $\mathrm{kHz}$, even when separated by hundreds of nanometers.

- AOTF has high efficiency. AOTF is highly efficient with transmission at the selected wavelength as high as $98 \%$. Unlike a "classical" monochromator in which the entrance/exit slits define the spectral resolution and limit the overall optical throughput, the spectral resolution of an AOTF is independent of the optical aperture. Therefore, optical throughput can be high. High efficiency translates directly into lower operating power, higher sensitivity and faster data acquisition.

- AOTF can be easily calibrated. For a given device geometry, the transmitted wavelength is determined only by the frequency of the applied RF signal, which can be generated with digital precision. Thus, an AOTF based spectrometer can be easily self-calibrated by changing the RF frequency. Since fluorescent spectroscopy applications usually require measurements at multiple wavelengths, short and long term wavelength repeatability are highly advantageous. As an example, a typical bulk $\mathrm{TeO}_{2}$ AOTF has a wavelength repeatability error of less than $\sim 0.05 \mathrm{~nm}$.

- AOTF can be easily computer controlled/integrated. AOTF has a high degree of controllability or programmability. In AOTF, the RF synthesizer is interfaced directly to a microprocessor or computer. This enables an AOTF based spectrometer to be programmed to scan or access different wavelengths very rapidly, and even to change the output intensity at those wavelengths. In use, therefore, it is easily integrated into almost any computer controlled measurement system. 


\section{MECHANICAL ISSUES}

Traditional spectrometers require careful handling and frequent calibration. Because an AOTF is an all solid-state tunable filter with no moving parts, it is a compact and rugged device. Thus, it is relatively immune to orientation changes or even severe mechanical shock and vibrations. Just as important, short of actually breaking the device, vibrations and shocks will not affect the wavelength calibration or the alignment.

\section{TEMPERATURE ISSUES}

Standard AOTF devices are not able to withstand and operate in cryogenic temperature because of the thermal expansion mismatch that exists between the transducer and the AO crystal ${ }^{14}$. Thus, the technique for bonding the transducer to the AO crystal is crucial in constructing an $\mathrm{AO}$ device for low temperature operation ${ }^{15,16}$. In the existing AO device technology, the transducer is bonded to the AO crystal by the use of an adhesive layer. The bonding techniques fall into two groups. In the first group (hard bonding), rigid adhesive layers such as metals or epoxy resins are employed. One major problem with hard bonding is that it results in thermal stress concentrated at the bonding interface due to the large thermal expansion mismatch between the transducer and AO crystal. For AO devices with an X-cut $\mathrm{LiNbO}_{3}$ bonded on $\mathrm{TeO}_{2} \mathrm{AO}$ crystal, the thermal stress will fracture a hard-bonded device at a temperature no lower than $-100^{\circ} \mathrm{C}$. Also, driving the transducer with RF power makes the device more susceptible to fracture.

The second group (soft bonding) uses flexible adhesives, such as silicon-based RTV, as the bonding layers. A soft bonding layer is flexible from room temperature down to its glass-transition temperature $\mathrm{T}_{\mathrm{g}}$, and turns rigid at cryogenic temperatures below $\mathrm{T}_{\mathrm{g}}$ (typical $\mathrm{T}_{\mathrm{g}}$ for silicon based RTVs is $\sim-120^{\circ} \mathrm{C}$ ). However, the soft bonding comes with major disadvantages: 1) the device does not work at temperatures below $\mathrm{T}_{\mathrm{g}} \sim-120^{\circ} \mathrm{C}$; 2) the impedance of the bonding layer varies with temperature cycles at low temperatures, making the device characteristics unrepeatable; 3 ) and the bonding quality degrades with temperature cycles.

There is a great need for $\mathrm{AO}$ devices that operate reliably at cryogenic temperatures below $-100^{\circ} \mathrm{C}$. For space flight missions, it is highly desirable that $\mathrm{AO}$ devices survive in the temperature extremes. It is also desirable to operate the $\mathrm{AO}$ device at low temperatures so that the device-generated thermal radiation does not contribute adversely to detector noise.

\section{RADIATION ISSUES}

\section{Radiation Effects in Inorganic AO Devices}

Van Allen belt trapped protons and electrons as well as other high energy radiations encountered in space can cause significant damage to inorganic and organic/ polymer AO and EO devices. Radiation hardened electronic devices have been constantly developed and improved for several decades and are successfully used in space systems. To some extent this is also true for commercially available inorganic AO devices, such as AOTFs, AO modulators and AO deflectors.

Over the period of 1991-1997, Taylor and colleagues first and extensively investigated and reported the passive and in situ radiation-induced responses exhibited by a variety of commercially available AO modulators and deflectors composed of lead molybdate $\left(\mathrm{PbMoO}_{4}\right)$, gallium phosphide $(\mathrm{GaP})$, indium phosphide $(\mathrm{InP})$, tellurium dioxide $\left(\mathrm{TeO}_{2}\right)$

and lithium niobate ${ }^{17-22}$. The devices were packaged but irradiated with the majority of metallic packaging removed. Gamma-ray, X-ray, electrons, proton and neutron irradiations were conducted to bound, delineate, and differentiate radiation-induced changes to operational Bragg modulators and deflectors. The majority of the irradiations were performed in situ, wherein the AO devices were fully operational during the irradiations and instantaneous changes to the AO device parameters such as amplitudes and shifts of spatial intensities, deflection angles, bandwidth, material absorption, optical transmission, diffraction efficiency, refractive index and polarization degree and states were quantized. Post-irradiation recovery of the radiation-induced changes was temporally measured and several perspicuous 
models for explaining and predicting the device degradation and recovery mechanisms from damage kinetics were developed. The device degradations were shown to primarily arise from the ionization and displacement induced formation of color centers and heating from the irradiation process. Depending on the electron or proton flux, radiationinduced heating resulted in the generation of temperature gradients within the $\mathrm{AO}$ crystalline materials, heat sinks and transducers contributing significantly to the degradation processes via alteration of the refractive index and sonic material sound velocity.

Shown in Figure 1 is the in-situ experimental arrangement for measuring proton and electron induced changes to diffraction efficiency and polarization changes in $\mathrm{TiO}_{2}$ and $\mathrm{GaP} \mathrm{AO}$ devices.

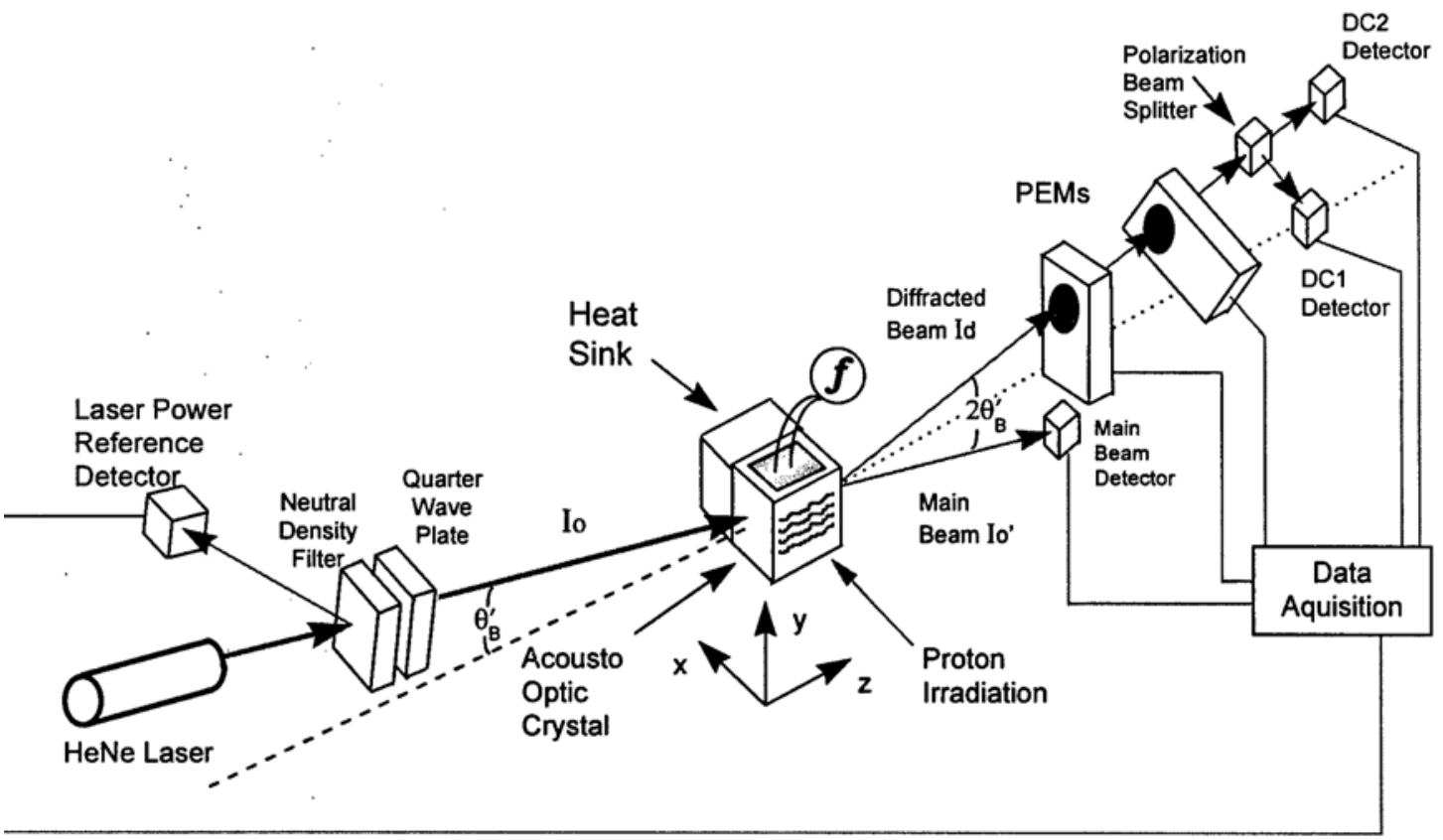

Figure 1. Measurement of high energy proton induced changes to AO device polarization and diffraction efficiency. Here $\mathrm{I}_{\mathrm{o}}$ is the incident beam to the AO device while $\mathrm{I}_{\mathrm{d}}$ and $\mathrm{I}_{0}$ represent the diffracted and throughput beams. Photoelastic modulators (PEMs) were used to measure the polarization changes ${ }^{21}$.

Figures 2 and 3 exemplify a few of the possible radiation induced effects generated in AO devices by high flux electrons and protons. High energy proton studies were conducted using the isochronous cyclotron of the Crocker Nuclear Laboratory, University of California, Davis, CA, while electron irradiations were conducted at the Army White Sands Missile Range, NM, linear electron accelerator facility. A full description of the experiments carried out at these facilities as well as other facilities for neutron, X-ray and gamma-irradiations can be found in Reference 21. 


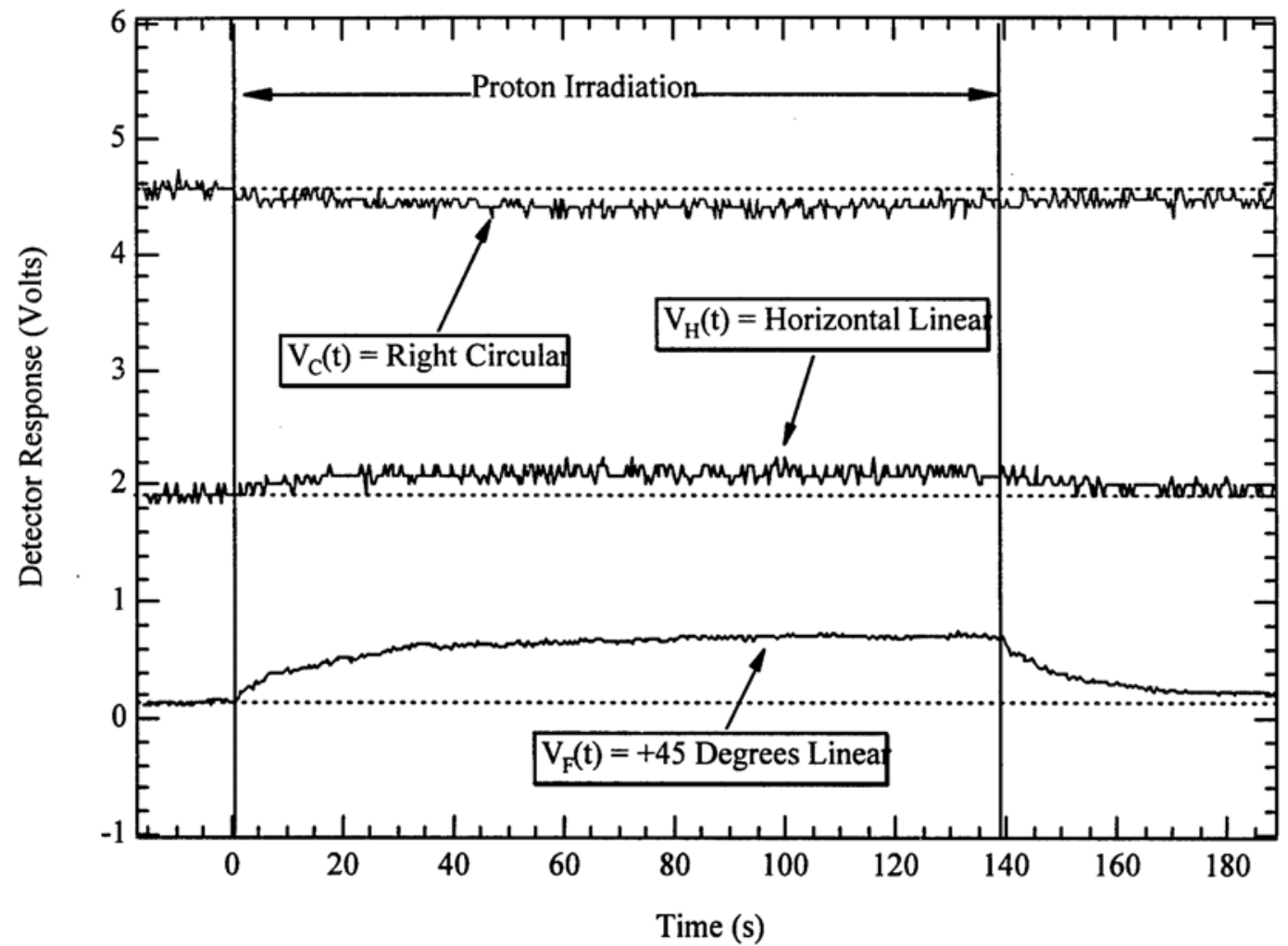

Figure 2. In situ proton irradiation $\left(\mathrm{E}_{\mathrm{p}}=39.9 \mathrm{MeV}\right)$ of a $\mathrm{TeO}_{2} \mathrm{AO}$ Bragg deflection device operating at $\lambda=6326 \mathrm{~nm}$, showing protoninduced transient changes to the diffracted beam polarization states. The proton fluence and flux were: $2.622 \times 10^{12} \mathrm{p} / \mathrm{cm}^{2}$ and $1.897 \mathrm{x}$ $10^{10} \mathrm{p} /\left(\mathrm{cm}^{2} \cdot \mathrm{s}\right)$, respectively. The irradiation time was $138.2 \mathrm{~s}$ (indicated by the time interval between the vertical lines) ${ }^{20,21}$.

Figure 1 shows the first reported proton-induced transient changes to polarization states in a $\mathrm{TeO}_{2} \mathrm{AO}$ deflector operated in its shear wave mode. Similar effects were reported for a GaP AO device for protons and electrons ${ }^{20,21}$. The right circular component decreases while the +45 degree linear and horizontal components increase in magnitude. Following cessation of the radiation (at $\mathrm{t}=138.2 \mathrm{~s}$ ) all components are nearly restored to their pre-irradiation magnitudes although at different times. The depolarization effects are attributed to proton induced heating $\left(\sim 0.8^{\circ} \mathrm{C}\right)$ of the $\mathrm{TeO}_{2}$ material resulting in temperature gradients, which in turn altered the crystal birefringence. Electron-induced depolarization is more pronounced and discussed elsewhere ${ }^{21}$. In all cases where AO crystal heating is present, the diffraction efficiency is affected (i.e. decreased) since different light polarizations will experience different elastooptic contributions to the refractive index changes. Moreover, significant heating and thermal gradients also affect the material sound velocity and hence the Bragg angle, as well as the sound-light interaction geometry since the phase homogeneity of the acoustic wavefronts is degraded. Using Stokes analysis for the data in Figure 2, the device pre-irradiation average degree of polarization $\left(\boldsymbol{P}_{\text {avg }}\right)$ at $\mathrm{t}<0$ s was calculated to be $\boldsymbol{P}_{\text {avg }}=96.47 \%$, while the maximum polarization decrease during the irradiation was calculated as $\boldsymbol{P}=93.7 \%$ at $\mathrm{t}=134 \mathrm{~s}$. No permanent depolarization was observed as a result of the applied proton fluence.

Shown in Figure 3 are the bandwidth responses for a $\mathrm{PbMoO}_{4} \mathrm{AO}$ deflector $(\lambda=6328 \mathrm{~nm})$ irradiated by $15 \mathrm{MeV}$ pulsed electrons. The device was operated at a center frequency, $f_{0}=80 \mathrm{MHz}$ and at a fixed Bragg angle (in air) of $0.40^{\circ}$. Normally, non-irradiated bandwidth measurements are performed under the reasonable assumption that $\mathrm{I}_{0}^{\prime}$ remains invariant. 


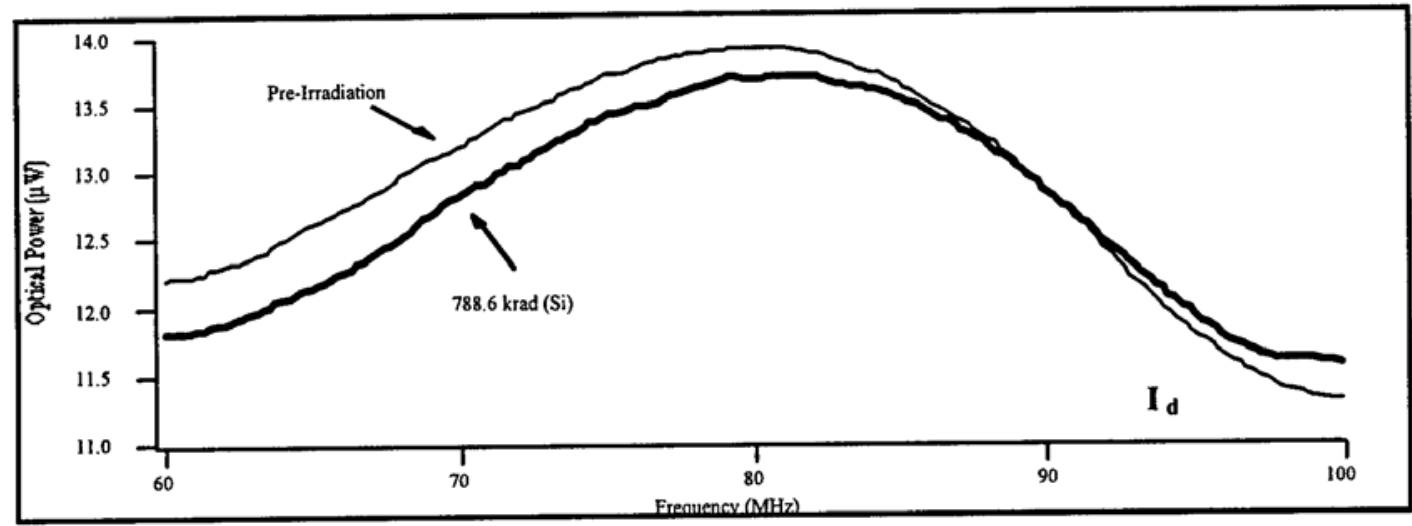

a.

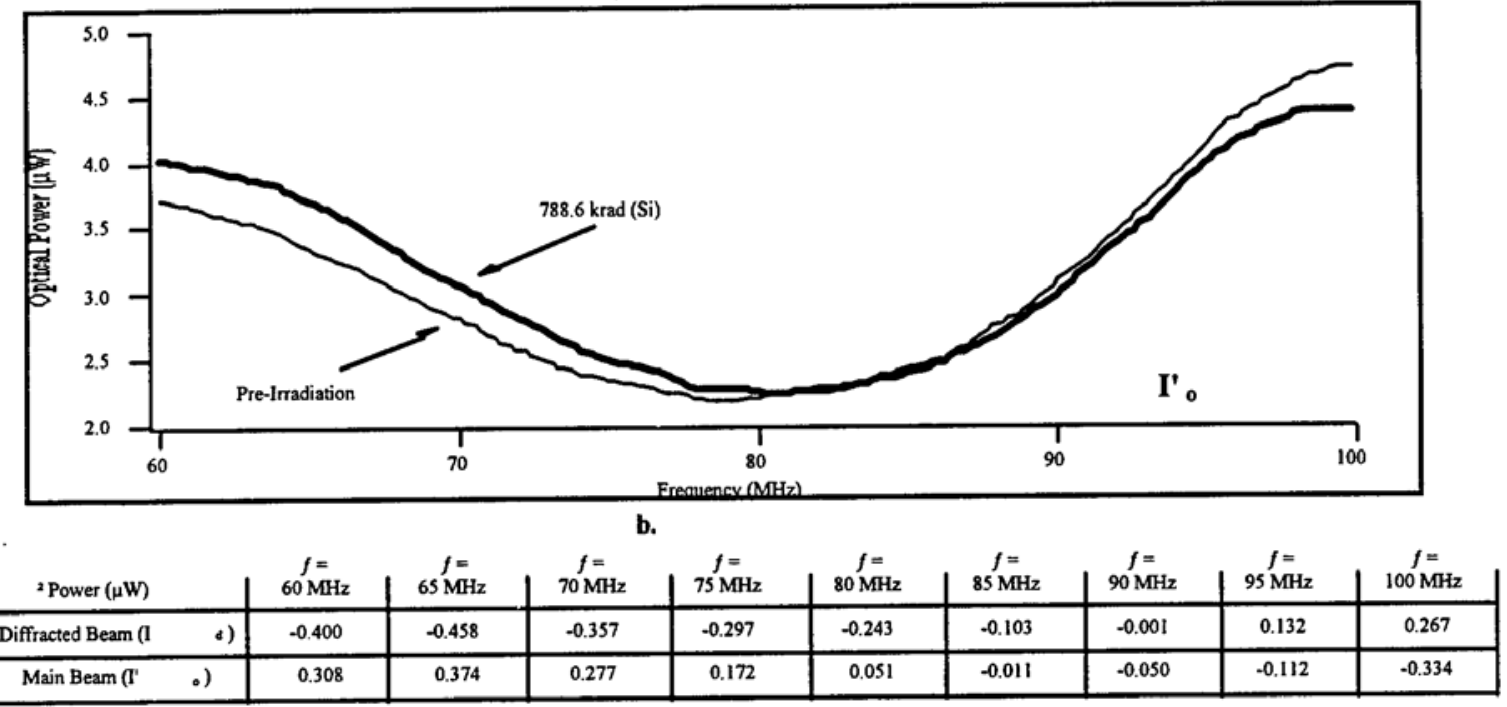

Figure 3. $15 \mathrm{MeV}$ electron induced bandwidth responses in a $\mathrm{PbMoO}_{4} \mathrm{AO}$ deflector. $\mathrm{I}_{\mathrm{d}}$ is the diffracted beam while $\mathrm{I}_{0}$ is the throughput beam shown in Figure 1. The swept frequency response was acquired after some 750 electron pulses resulting in a total dose of $788.6 \mathrm{krad}(\mathrm{Si})^{20,21}$.

As first shown in Figure 3, correlated energy exchanges between both $\mathrm{I}_{\mathrm{d}}$ and $\mathrm{I}_{0}$ occur during the electron irradiations. Generally, the power in I' (throughput) beam was observed to increase while the power correspondingly decreased in the diffracted beam as the number of pulses increased and the dose accumulated. However, this process appears to reverse at the far end of the frequency. This is attributed to decoupling of the acouto-optic interaction resulting from the phase inhomogeneity of the acoustic wavefronts. The temperature coefficient of index for $\mathrm{PbMoO}_{4}$ is given by the expression

$$
\mathrm{dn} / \mathrm{dT}=[(1 / \mathrm{n}) /(\mathrm{dn} / \mathrm{dT})]
$$

and is negative since $\mathrm{dn} / \mathrm{dT}=-71.58 \times 10^{-6}{ }^{\circ} \mathrm{C}^{-1}$ for the ordinary ray direction. This would indicate that the refractive index of the crystal medium is lowered due to electron induced volumetric heating, consistent with the heating caused response data shown in Figure 3. Permanent attenuation of the spatial intensities over the bandwidth studied did not occur $^{21}$. 
The data substantiates that $\mathrm{AO}$ devices are susceptible to energetic protons and electrons (and other radiations discussed in reference 21) under high flux conditions and to high total dose. However, these conditions are orders of magnitude higher than space radiation fluxes and doses found in near- Earth orbit. Thus the early AFRL study concluded that AO Bragg deflectors and modulators are quite insensitive to the long term, low flux radiation environments experienced in the near-Earth space environment ${ }^{21}$.

Economical gamma-rays are often used to simulate the total dose received by materials and devices in space. Use of $\mathrm{a}^{60} \mathrm{Co}$ gamma-ray source results in photons with predominant energies of 1.17 and $1.332 \mathrm{MeV}$, and are far less than electron and proton energies found in space that range from a few $\mathrm{keV}$ to 100 's of $\mathrm{MeV}$. Unlike protons, electrons and other energetic ions comprising the space environment, irradiation of the AO materials and devices by energetic photons (gamma-rays) produces far less damage in the afore-mentioned irradiated $\mathrm{AO}$ materials and devices ${ }^{22}$.

However, damage by massive doses of gamma-rays is possible. For example, passive gamma-ray irradiation of AO devices conducted by Soos resulted in permanent damage ${ }^{23}$.

In the study by Soos and colleagues, a GaP shear mode deflector, $\mathrm{TeO}_{2}$ shear mode deflector, and a $\mathrm{LiNbO}_{3}$ longitudinal mode deflector and a $\mathrm{TeO}_{2}$ acousto-optic tunable filter were gamma-ray irradiated. The transducer material used in all of these devices was $\mathrm{LiNbO}_{3}$. The $\mathrm{AO}$ devices were gamma-ray irradiated to a dose of $1 \mathrm{Mrad}$ using a ${ }^{60} \mathrm{Co}$ source. The devices were tested in the form that they would be used in a system, and consisted of the AO crystal, piezoelectric transducer, aluminum housing, RF connector and a strip line with RF matching components. The following properties were measured: optical transmission, diffraction efficiency vs. RF power, diffraction efficiency vs distance along the optical aperture, acoustic velocity, diffraction efficiency vs. frequency, and electrical properties.

Only small changes were observed in the optical transmission of the AOTF following gamma-ray exposure. Table 1 summarizes these results (incident angle was set at $90 \mathrm{deg}$.

Table 1. Optical transmission of $\mathrm{TeO}_{2}$ AOTF before and after gamma-ray irradiation to a dose of $1 \mathrm{Mrad}^{23}$.

\begin{tabular}{|c|c|c|}
\hline \multirow{2}{*}{$\begin{array}{c}\text { Distance from transducer } \\
(\mathrm{mm})\end{array}$} & \multicolumn{2}{|c|}{ \% Optical Transmission } \\
\cline { 2 - 3 } & Before Radiation & After radiation \\
\hline 0 & $97 \pm 0.5$ & $95 \pm 0.5$ \\
\hline 1 & $98 \pm 0.5$ & $96 \pm 0.5$ \\
\hline 2 & $98 \pm 0.5$ & $95 \pm 0.5$ \\
\hline 3 & $98 \pm 0.5$ & $93 \pm 0.5$ \\
\hline
\end{tabular}

The change of diffraction efficiency vs power was measured after exposure of the $\mathrm{TeO}_{2}$ shear mode at a constant frequency of $130 \mathrm{MHz}$. The rate of change of the diffraction efficiency with RF power was the same before and after gamma-ray exposure.

Table 2 shows the results of the change of diffraction efficiency along the optical aperture of the $\mathrm{TeO}_{2} \mathrm{AOTF}$. The difference in these measurements before and after radiation exposure is within the error of measurement.

Table 2. Diffraction efficiency vs distance along the AOTF optical aperture [23].

\begin{tabular}{|c|c|c|}
\hline \multirow{2}{*}{$\begin{array}{c}\text { Distance from transducer } \\
(\mathrm{mm})\end{array}$} & \multicolumn{2}{|c|}{ \% Diffraction Efficiency } \\
\cline { 2 - 3 } & Before Irradiation & After gamma-ray irradiation \\
\hline 0 & $94 \pm 0.5$ & $92 \pm 0.5$ \\
\hline 1 & $88 \pm 0.5$ & $92 \pm 0.5$ \\
\hline 2 & $85 \pm 0.5$ & $90 \pm 0.5$ \\
\hline 3 & $88 \pm 0.5$ & $84 \pm 0.5$ \\
\hline
\end{tabular}


Based on separation angle test results, there is no visible change in $\mathrm{TeO}_{2}$ birefringence value due to exposure by gammarays. Also, there were no observable permanent changes in the polarization direction as a result of the exposure.

The $\mathrm{TeO}_{2} \mathrm{AOTF}$ diffraction efficiency vs. RF frequency characteristics before and after gamma-ray exposure were measured. These curves showed the optical bandpass of the AOTF as well as the RF frequency-wavelength relation. The results indicated that the bandpass of the AOTF changed after exposure from $1.282 \mathrm{~nm}$ to $1.734 \mathrm{~nm}$ and that the RF frequency-wavelength relation was shifted by $10 \mathrm{MHz}$. The $633 \mathrm{~nm}$ laser peak was at $123.4 \mathrm{MHz}$ before radiation exposure and than was shifted down to $113.14 \mathrm{MHz}$ after exposure.

\section{Radiation effects in Polymer EO Modulators}

More recently, Taylor and colleagues first investigated and reported the radiation resistance and responses of poledEO polymer modulators. In a 2003 investigation it was demonstrated that devices not strongly poled (therefore possessing large $\mathrm{V}$ values) were likely to de-pole when irradiated by gamma-rays ${ }^{11}$. In a subsequent investigation it was observed that some strongly poled devices actually experienced a substantial lowering of their $\mathrm{V}_{\pi}$ values when irradiated by gamma-rays over a low dose range ${ }^{8}$.

Shown in Figure 4 is the $\mathrm{V}_{\pi}$ response of several Mach-Zehnder EO modulators comprised of a guest-chromophore (phenyltetrane)- and host- polymer (amorphous polycarbonate) irradiated by ${ }^{60} \mathrm{Co}$ gamma-rays at Sandia National Laboratory and irradiated by $25.6 \mathrm{MeV}$ protons using the isochronous cyclotron located at Crocker Nuclear Laboratory $(\mathrm{CNL})^{8}$. Figure 4 shows that $\mathrm{V}_{\pi}$ decreased following irradiation by gamma-rays and is only slightly altered by proton irradiations. The process believed responsible for the $\Delta \mathrm{V}_{\pi}$ responses may be similar to refractive index increases of $\sim 10^{-}$ ${ }^{2}-10^{-3}$ reported for irradiated polymethyl methacrylate (PMMA) arising from free volume changes that can increase the modulator core refractive index. For example ${ }^{8}$, since $\mathrm{V}_{\pi}$ is proportional to the refractive index $\left(\mathrm{V}_{\pi}=\lambda \mathrm{d} / \mathrm{r}_{33} \mathrm{n}^{3} \Gamma \mathrm{L}\right)$, a change of index corresponding to $\Delta n=0.03$ would be required to result in $\Delta \mathrm{V}_{\pi}$ reduction of $6 \%$ for a device having a $\mathrm{V}_{\pi}$ of $6 \mathrm{~V}$, electrode length (L) of $2 \mathrm{~cm}$, EO coefficient of $\mathrm{r}_{33}=24 \mathrm{pm} / \mathrm{V}, \mathrm{n}$ (core) $=1.62$, device thickness (d) $=7.9 \mu \mathrm{m}$ and $\lambda=1$. The data shown in Figure 5 indicate that properly designed polymer modulators have a high potential for space applications.

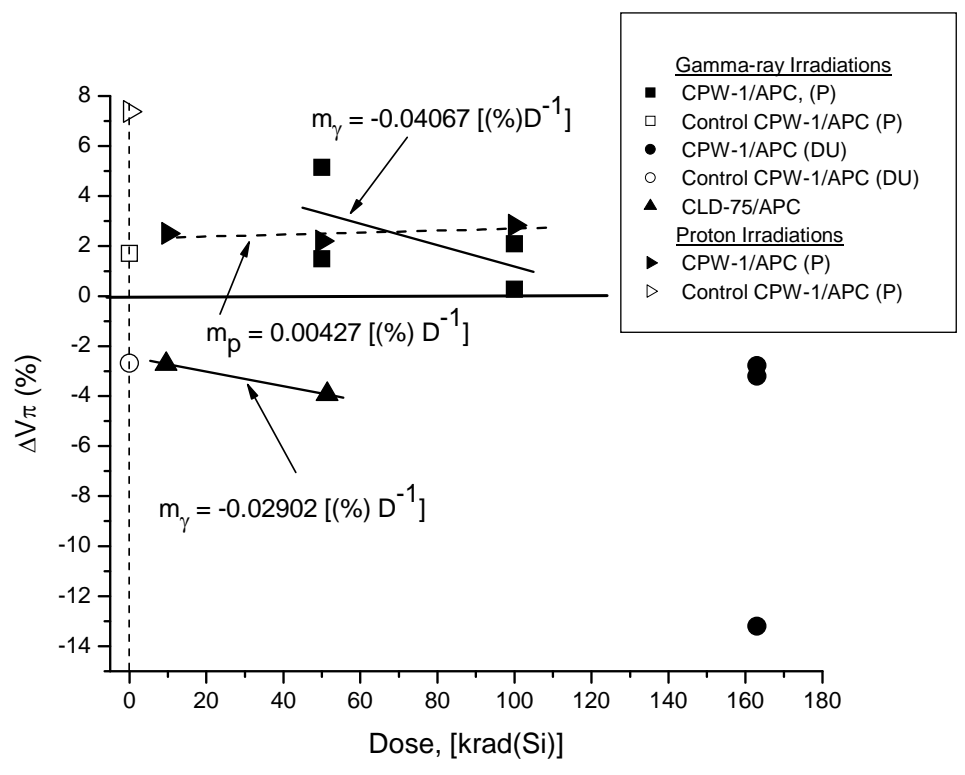

Figure 4. Changes in irradiated polymer modulator switching voltage $\left(\mathrm{V}_{\pi}\right)$. The responses are normalized to a $1 \mathrm{~cm}$ interaction length. The slope $(\mathrm{m})$ values indicate linear scaling of the switching voltage among some devices irradiated to a total dose of $100 \mathrm{krad}(\mathrm{Si})^{8}$. 


\section{CONCLUSIONS}

In this paper we discussed the major space qualification issues for acousto- and electro-optical devices. Acoustoand electro-optical devices form the major components of many current space based optical systems. The major space qualification issues are related to: mechanical stability, thermal effects and operation of the devices in the naturally occurring space radiation environment. We discussed the stability of acousto- and electro-optic materials and devices against mechanical vibrations, thermal cycling in operating and non-operating conditions and device responses to space ionizing and displacement radiation effects.

\section{REFERENCES}

1. N. Narasimhan, D.R. Patel, "Technological Advances in Space Simulation Techniques", http://www.ipr.res.in/ivsns05/manuscript/IT7.pdf.

2. D. S. McKnight, R. E. Dueber, E. W. Taylor, "Space Debris and Micrometeorite Events Experienced by WL Exp \#701 In Prolonged Low-Earth Orbit,” Jour. Geophysical Research - Space Physics, 96, No. A6, June 1991.

3. T. B. McCord et al. "Salt on Europa's Surface Detected by Galileo's Near Infrared Mapping Spectrometer", Science, vol 280, p1242(1998).

4. H. Zhang, X.L. Wang, J.I. Soos and J.A. Crisp. Design of a miniature solid state NIR spectrometer. Proc. SPIE, 2475, pp 376-383, 1995.

5. O. Korablev, J.L. Bertaux, E. Dimarellis, A. Grigoriev, Yu. Kalinnikov, A. Stepanov and S. Guibert. An AOTFbased spectrometer for MARS atmosphere sounding. Proc. SPIE, 4818, pp. 261-271 (2002).

6. O. Korablev, J.L. Bertaux, A. Grigoriev, E. Dimarellis, Yu. Kalinnikov, A. Rodin, C. Muller, D. Fonteyn. An AOTF-based spectrometer for the studies of Mars atmosphere for MARS express ESA mission. Advances in Space Research, Volume 29, Issue 2, p. 143-150 (2002).

7. J. Chance Carter, Jon Scaffidi, Shana Burnett, Bryan Vasser, Shiv K. Sharma and S. Michael Angel. Stand-off Raman Detection using dispersive and tunable filter based systems. Spectrochimica Acta Part A 61 pp. 2288-2298 (2005).

8. E. W. Taylor, J. E. Nichter, F. Nash, F. Haas, A. A. Szep, R. J. Michalak, B. M. Flusche, P. Payson, G. A.. Brost, A. R. Pirich,' C. Castaneda, B. Tsap, H. R. Fetterman, "Radiation resistance of electro-optic polymer based modulators", Applied Physics Letters, 86, May 2005.

9. E. W. Taylor, "Interaction and responses of linear and nonlinear optic polymer based photonic materials and devices with ionizing radiation", Proc. SPIE, Vol. 5897, August 2005.

10. E. W. Taylor J. Nichter, F. Nash, F. Haas, A. A. Szep, R. J. Michalak, B. M. Flusche, P. Repak, G. Brost, A. Pirich, D. Craig D. Le, D. Cardimona, H. R. Fetterman, B. Tsap, C. Castaneda, R. Barto, T. Zeng, D. Wood, R. O. Claus, "Radiation resistant polymer-based photonics for space applications" Proc SPIE, Vol. 5554, August 2004.

11. E. W. Taylor, J. Nichter, F. Nash, R. Michalak, F. Haas, P. Payson, P. Cook, T. McEwen, B. McKeon, A. Szep, B. Flushe, A. Pirich, G. Brost, J. Grote, J. Zetts, P. Yaney, E. Heckman "Behavior of NLO Polymer Modulators Irradiated by Gamma-Rays", Proc. SPIE, Vol. 5212, 7 August 2003.

12. E. W. Taylor, "Inorganic and Polymer Photonic Sensor Technologies in Space Missions" (Invited Experts Lecture) Proc. $18^{\text {th }}$ IEEE Instrumentation and Measurement Conference, Univ. Budapest, Budapest, Hungary, 21-23 May 2001.

13. I.C. Chang, Tunable acousto-optic filters: An overview. Opt. Eng., 16:5 pp.455-460, 1977.

14. S. Kim and H. Ledbetter, " Low temperature elastic coefficients of polycrystalline indium", Materials Science \& Engineering, Vol. A252, P31 (1998).

15. J. Xu and R. Stroud, Acousto-Optic Devices, (Jon Wiley \& Sons, Inc., New York, 1992).

16. M. Khoshnevisan et al, "Development of Cryogenic Infrared Acousto-optic Tunable Filter", SPIE Vol 245, p63 (1980).

17. E. W. Taylor, A. D. Sanchez, S. A. DeWalt, et. al., "Radiation Induced Effects in Acousto Optic Devices", SPIE Integrated Optics Conference - Guided Wave Radiation Effects, SPIE Proc., 1794, 217-224, September 1992.

18. E. W. Taylor, S. P. Chapman, M. A. Kelly, A. D. Sanchez, J. Stohs, E. Kinsley, D. M. Craig, "Radiation Induced Bandwidth Responses in an Acousto Optic Bragg Cell”, SPIE Proc., 2482, 19 -20 April 1995. 
19. H. Paxton, E. W. Taylor, "Thermal Models for Laser Heating of Acousto Optic Devices" SPIE Proc., 2811, August, 96.

20. E. W. Taylor, J. E. Winter, A. D. Sanchez, S. J. McKinney "Proton Induced Transient Depolarization in Gallium Phosphide and Tellurium Dioxide Bragg Cells", J. Opt. Engr., 36, No. 7, July 97.

21. E. W. Taylor, A. D. Sanchez, J. E. Winter, S. J. McKinney, A. H. Paxton, H. Schone, D. P. Craig, A Study of Radiation Induced Effects in PhotonicDevices: Acousto Optic Modulators and Deflectors, AFRL-VS-TR-19981028.

22. E. W. Taylor, Radiation Effects, Chapter 14.1 in: Properties of Lithium Niobate and Other Novel Ferroelectric Materials, Editor, K.K. Wong, INSPEC Pub., IEEMIS Datareview Series No. 28, UK, July 2002.

23. Jolanta Soos, Sean Wang, Robert Scheerer. "Nuclear Hardening and survivability of acousto-optics" Final Report for Phase I contract \#DNA 001-93-C-0132, sponsored by the Defense Nuclear Agency. Completed Feb. 1996. 